\title{
The maximum number of cycles in a graph with fixed number of edges
}

\author{
Andrii Arman \\ School of Mathematics \\ Monash University \\ Melbourne, Australia \\ andrii.arman@monash.edu
}

\author{
Sergei Tsaturian \\ Department of Mathematics \\ University of Manitoba \\ Winnipeg, Canada \\ s.tsaturian@gmail.com
}

Submitted: May 20, 2019; Accepted: Nov 6, 2019; Published: Dec 6, 2019

(C) The authors. Released under the CC BY-ND license (International 4.0).

\begin{abstract}
The main problem considered in this paper is maximizing the number of cycles in a graph with given number of edges. In 2009, Király conjectured that there is constant $c$ such that any graph with $m$ edges has at most $c(1.4)^{m}$ cycles. In this paper, it is shown that for sufficiently large $m$, a graph with $m$ edges has at most $(1.443)^{m}$ cycles. For sufficiently large $m$, examples of a graph with $m$ edges and $(1.37)^{m}$ cycles are presented. For a graph with given number of vertices and edges an upper bound on the maximal number of cycles is given. Also, bounds tight up to a constant are presented for the maximum number of cycles in a multigraph with given number of edges, as well as in a multigraph with given number of vertices and edges.
\end{abstract}

Mathematics Subject Classifications: 05C35, 05C38

\section{Introduction}

Counting the number of cycles in a graph is a problem that was studied for different classes of the graphs: graphs with given cyclomatic number, planar graphs, 3-regular and 4-regular graphs, and many others. However, only a few general bounds for the number of cycles that use basic graph parameters are known. In this paper, bounds on the number of cycles in a graph as a function of the number of vertices and edges are presented.

Let $C(G)$ be the number of cycles in a graph $G$. In 1897, Ahrens [1] proved that for a graph $G$ with $n$ vertices, $m$ edges and $k$ components,

$$
m-n+k \leqslant C(G) \leqslant 2^{m-n+k}-1 .
$$

The lower bound in (1) is tight; for example, it is achieved by any disjoint union of cycles and trees. The tightness of the upper bound in (1) was shown by Mateti and Deo 
[10] and the only graphs for which the upper bound is tight are the ones that can be obtained from subdivisions of $K_{3}, K_{4}, K_{3,3}$ and $K_{4}-e$ by sequentially adding pendant edges. Aldred and Thomassen [2] improved the upper bound in (1) by showing that for a connected graph $G$,

$$
C(G) \leqslant \frac{15}{16} 2^{m-n+1} .
$$

Entringer and Slater [7] considered $C(G)$ for the class of connected graphs with fixed cyclomatic number $r=m-n+1$. It follows from the results of [7] that there is a 3regular connected graph $G$ for which $C(G)>2^{r-1}$. Shi [13] presented an example of an outer-planar 3-regular Hamiltonian graph $G$ with $C(G)=2^{r-1}+r-1$.

Alt, Fuchs and Kriegel [3] and Aldred and Thomassen [2] studied $C(G)$ for the class of planar graphs, in terms of the number of vertices and in terms of the cyclomatic number, respectively. Arman, Gunderson and Tsaturian [5] studied $C(G)$ for the class of trianglefree graphs on $n$ vertices, and recently, Morrison, Roberts and Scott [11] studied $C(G)$ for the class of $H$-free graphs, where $H$ contains a colour-critical edge.

Király [9] investigated $C(G)$ for several classes of graphs: the union and the sum of two trees, 3-regular and 4-regular graphs, and graphs with average degree 4 . Király also conjectured that there is a constant $c$, such that for any graph $G$ that has $m$ edges,

$$
C(G) \leqslant c(1.4)^{m}
$$

We refer an interested reader to $[4,5,14]$ for a more comprehensive literature review.

Motivated by Ahrens's bound (1) and by the work of Király, in this paper we further investigate $C(G)$ for two classes of graphs: those with $n$ vertices and $m$ edges, and those with $m$ edges.

The main result of this paper is providing an estimate on $C(G)$ for graphs with given number of vertices and edges.

Theorem 1. Let $G$ be a multigraph with $n \geqslant 2$ vertices and $m$ edges. If $\frac{m}{n-1}<3$, then

$$
C(G)<\frac{3}{4} \Delta(G) \cdot(\sqrt[3]{3})^{m} .
$$

If $\frac{m}{n-1} \geqslant 3$, and $\left\lfloor\frac{m}{n-1}\right\rfloor=s, \alpha=\frac{m}{n-1}-s$, then

$$
C(G)<\frac{3}{4} \Delta(G)\left(s^{1-\alpha}(s+1)^{\alpha}\right)^{n-1} .
$$

A more compact, but slightly weaker bounds can be obtained as a corollary of Theorem 1:

$$
C(G) \leqslant\left\{\begin{array}{l}
\frac{3}{4} \Delta(G)\left(\frac{m}{n-1}\right)^{n-1}, \text { if } \frac{m}{n-1} \geqslant 3 \\
\frac{3}{4} \Delta(G) \cdot(\sqrt[3]{3})^{m}, \text { if } \frac{m}{n-1}<3
\end{array}\right.
$$


The bound in (3) is better than the one in (2) for graphs with sufficiently large number of edges and average degree at least 4.25. The main tool used to derive Theorem 1 is Lemma 4 that provides an estimate on the number of cycles that contain a given path.

For $m \in \mathbb{Z}^{+}$let $C(m)$ be the maximum number of cycles in a graph with $m$ edges. As a consequence of Theorem 1 we also obtain a bound for $C(m)$.

Corollary 2. For any positive integer $m$,

$$
C(m)<4.5(\sqrt[3]{3})^{m}
$$

Theorem 1 and Corollary 2 are proved in Section 2 .

In Section 3, for $m$ sufficiently large, a graph $G$ with $m$ edges is constructed, such that

$$
C(G) \geqslant(2+\sqrt{8})^{\frac{m}{5}-1} \geqslant 1.37^{m} .
$$

Corollary 2 and the result of Section 3 imply that for $m$ large enough,

$$
1.37^{m} \leqslant C(m) \leqslant 1.443^{m} .
$$

The lower and upper bound in (4) differ exponentially, which motivated us to question the sharpness of Lemma 4. Although this lemma might not be sharp for counting cycles in graphs, we show in Section 4 that Lemma 4 provides exponentially sharp bound on the number of cycles in multigraphs. It is shown (Theorem 8) that if $G$ is a multigraph that has the most cycles among all loopless multigraphs with $m$ edges, then

$$
\frac{9}{10}(\sqrt[3]{3})^{m} \leqslant C(G) \leqslant 4.5(\sqrt[3]{3})^{m}
$$

Finally, in Appendix $\mathrm{A}$ it is shown that extremal graphs for the function $C(m)$ have bounded degrees. Namely, it is shown (Theorem 10) that if $G$ is a graph with $m$ edges with $C(G)=C(m)$, then the maximum degree of $G$ is at most 11 .

\section{Main results}

For $k \in \mathbb{Z}^{+}$, denote $\{i \in \mathbb{Z} ; 1 \leqslant i \leqslant k\}$ by $[k]$, and for a set $S$, denote $\{T \subseteq S:|T|=k\}$ by $[S]^{k}$. A multigraph in this paper is an ordered triplet $G=(V, E, t)$, where $V$ is a set of vertices, $E$ is a set of edges and $t$ is a function that maps $E$ to $[V]^{2}(t(e)$ indicates which vertices are incident to $e \in E)$. Note that under this notation the edge set of a multigraph is indeed a set (not a multiset). The degree $\operatorname{deg}_{G}(V)$ of a vertex $v \in V(G)$ is the number of edges incident to $v$. For two vertices $u, v \in V(G)$, denote by $E(u, v)$ the set of all edges between $u$ and $v$. For a vertex $v \in V(G)$, denote by $N(v)$ the set of all vertices connected with $v$ by at least one edge. Denote the average degree of a graph (or a multigraph) $G$ by $d(G)$, the maximum degree by $\Delta(G)$, and the minimum degree by $\delta(G)$. A cycle in a multigraph $G$ is a set of $k \geqslant 2$ distinct vertices and $k$ distinct edges $\left\{v_{1}, e_{1}, v_{2}, e_{2}, \ldots, e_{k}, v_{1}\right\}$, where for each $i \in[k], v_{i} \in V(G), e_{i} \in E(G)$ and any consecutive 
vertex and edge are incident. As in the case of simple graphs, denote the number of cycles in a multigraph $G$ by $C(G)$.

In order to prove Theorem 1, we introduce a counting Lemma 4 that bounds the number of cycles in a multigraph through a given path by essentially a number of ways to extend that path to a longer one. Lemma 4 is subsequently used to obtain Lemma 5 which provides an estimate on the number of cycles through a given vertex in a graph. We then use Lemma 5 in combination with induction to prove Theorem 1. Finally, we establish Corollary 2 as a quick consequence of Theorem 1.

Definitions 3. Let $G$ be a multigraph with $n$ vertices.

Let $P=v_{1} e_{1} v_{2} \ldots e_{k-1} v_{k}$ be a path in $G$. Define

$$
f(P)=\max \left\{\operatorname{deg}_{G-\left\{v_{2}, \ldots, v_{k-1}\right\}}\left(v_{k}\right), 1\right\},
$$

i.e. $f(P)$ is equal to the number of ways to select the next vertex on a cycle that starts with $P$, or equal to 1 if $P$ cannot be extended to a cycle. Denote the number of cycles in $G$ that contain $P$ by $C(P)$.

For two paths $P_{1}$ and $P_{2}$ with the same starting vertex, write $P_{1} \subseteq P_{2}$ if $P_{1}$ is a subpath of $P_{2}$ (i.e. $\left.P_{1}=v_{1} e_{1} v_{2} \ldots e_{k-1} v_{k}, P_{2}=v_{1} e_{1} v_{2} \ldots e_{k-1} v_{k} e_{k} \ldots e_{\ell-1} v_{\ell}\right)$. We write $P_{1} \subset P_{2}$ if $P_{1} \subseteq P_{2}$ and $P_{1} \neq P_{2}$. In this case, define $f\left(P_{1}, P_{2}\right)=\prod_{j=k}^{\ell} f\left(v_{1} e_{1} \ldots v_{j}\right)$.

Lemma 4. Let $G$ be a multigraph with $n \geqslant 2$ vertices, $k \in[n]$, and $P=v_{1} e_{1} v_{2} e_{2} \ldots v_{k}$ be a path in $G$. Then

$$
C(P) \leqslant \max _{P^{\prime}: P \subseteq P^{\prime}} f\left(P, P^{\prime}\right) .
$$

Proof. For a path $P=v_{1} e_{1} v_{2} \ldots e_{k-1} v_{k}$ additionally define

$$
F(P)=\left\{P^{\prime}: P^{\prime} \text { is a path of form } P e_{k} v_{k+1}\right\},
$$

i.e. $F(P)$ is the set of paths that extend $P$ by exactly one edge.

Fix $n \geqslant 2$. Let $G$ be a multigraph on $n$ vertices. The proof is by downwards induction on $k$.

Base case. Let $k=n$. Let $P=v_{1} e_{1} \ldots v_{n}$ be a path in $G, C(P)$ in this case is equal to the number of edges between $v_{n}$ and $v_{1}$ and $\max _{P \subseteq P^{\prime}} f\left(P, P^{\prime}\right)=f(P) . f(P)$ is at least the number of edges between $v_{n}$ and $v_{1}$, hence this proves the base case.

Inductive step. Let $\ell \in[n-1]$. Assume that the statement of the lemma holds for $k=\ell+1$, and prove it for $\ell$, i.e. let $P=v_{1} e_{1} \ldots v_{\ell}$, and we seek to bound $C(P)$.

Let $s$ be the number of edges between $v_{\ell}$ and $v_{1}$. Then

$$
C(P)=s+\sum_{P^{\prime} \in F(P)} C\left(P^{\prime}\right)
$$

If $F(P)=\emptyset$ then $C(P)=s \leqslant f(P)$ and hence the inductive step follows. Now assume that $F(P) \neq \emptyset$. For all possible choices of $P^{\prime} \in F(P)$, according to inductive hypothesis,

$$
C\left(P^{\prime}\right) \leqslant \max _{P^{\prime \prime}: P^{\prime} \subseteq P^{\prime \prime}} f\left(P^{\prime}, P^{\prime \prime}\right) .
$$


Therefore,

$$
\begin{aligned}
C(P) & \leqslant s+(f(P)-s) \max _{P^{\prime} \in F(P)} C\left(P^{\prime}\right) \\
& \leqslant s+(f(P)-s) \max _{P^{\prime} \in F(P)} \max _{P^{\prime \prime}: P^{\prime} \subseteq P^{\prime \prime}} f\left(P^{\prime}, P^{\prime \prime}\right) \\
& \leqslant f(P) \max _{P^{\prime} \in F(P)} \max _{P^{\prime \prime}: P^{\prime} \subseteq P^{\prime \prime}} f\left(P^{\prime}, P^{\prime \prime}\right) \\
& =\max _{P^{\prime} \in F(P)} \max _{P^{\prime \prime}: P^{\prime} \subseteq P^{\prime \prime}} f(P) f\left(P^{\prime}, P^{\prime \prime}\right) \\
& =\max _{P^{\prime} \in F(P)} \max _{P^{\prime \prime}: P^{\prime} \subseteq P^{\prime \prime}} f\left(P, P^{\prime \prime}\right)=\max _{P^{\prime \prime}: P \subseteq P^{\prime \prime}} f\left(P, P^{\prime \prime}\right) .
\end{aligned}
$$

This proves that the statement of the lemma holds for $\ell$, and therefore by induction it holds for all $k \in[n]$.

Lemma 5. Let $G$ be a multigraph with $n \geqslant 3$ vertices and $m$ edges, and let $v_{1}$ be a vertex in $G$ of degree $\Delta(G)$.

- If $\frac{m}{n-1} \geqslant 3$, and $\left\lfloor\frac{m}{n-1}\right\rfloor=s, \frac{m}{n-1}-s=\alpha$, then there are at most $\frac{\Delta(G)}{2}\left(s^{1-\alpha}(s+1)^{\alpha}\right)^{n-1}$ cycles in $G$ that contain $v_{1}$.

- If $\frac{m}{n-1}<3$, then there are at most $\frac{\Delta(G)}{2} \cdot(\sqrt[3]{3})^{m}$ cycles in $G$ that contain $v_{1}$.

Proof. Let $G$ be a multigraph with $n \geqslant 3$ vertices and $m$ edges, and $v_{1}$ be a vertex with degree $\Delta(G)$.

For any edge $e=v_{1} v_{2}$ incident to $v_{1}$, by Lemma 4 , the number of cycles that contain $e$ is at most

$$
\max _{P^{\prime}: e \subseteq P^{\prime}} f\left(e, P^{\prime}\right)
$$

Every cycle through $v_{1}$ contains two such edges, therefore the number of cycles that contain $v_{1}$ is at most

$$
\frac{\Delta}{2} \max _{e=v_{1} v_{2}} \max _{P^{\prime}: e \subseteq P^{\prime}} f\left(e, P^{\prime}\right) .
$$

Let $e=v_{1} v_{2}$ and $P^{\prime}=v_{1} v_{2}, \ldots v_{t}$ be an edge and a path that give the maximum in (5) with the smallest possible $t$. For $2 \leqslant i \leqslant t$ define $f_{i}=f\left(v_{1} \ldots v_{i}\right)$, so

$$
\frac{\Delta}{2} \max _{e=v_{1} v_{2}} \max _{P^{\prime}: e \subseteq P^{\prime}} f\left(e, P^{\prime}\right)=\frac{\Delta}{2} f_{2} \cdot \ldots \cdot f_{t} .
$$

Then $f_{t} \geqslant 2$ (otherwise remove all $f_{i}=1$ after the last $f_{k} \geqslant 2$ to obtain the smaller collection of vertices that gives maximum in (5)). Then for all $2 \leqslant i \leqslant t$,

$$
f_{i}=\operatorname{deg}_{G-\left\{v_{2}, \ldots, v_{i-1}\right\}}\left(v_{i}\right) .
$$

For $2 \leqslant i \leqslant t$, all the edge sets $\left\{v_{i} u \in E(G): u \in V(G) \backslash\left\{v_{2}, \ldots, v_{i}\right\}\right\}$ are mutually disjoint, so $f_{2}+\cdots+f_{t} \leqslant m$. Therefore,

$$
\frac{\Delta}{2} f_{2} \cdot \ldots \cdot f_{t} \leqslant \frac{\Delta}{2} \cdot \max _{\substack{2 \leqslant t \leqslant n \\ x_{2}+\ldots+x_{t} \leqslant m \\ \forall i \in[2, t], x_{i} \in \mathbb{Z}^{+}}}\left\{x_{2} \cdot x_{3} \cdot \ldots \cdot x_{t}\right\} .
$$


So the number of cycles in $G$ that contain $v_{1}$ is at most

$$
\frac{\Delta}{2} \cdot \max _{\substack{2 \leqslant t \leqslant n \\ x_{2}+\ldots+x_{t} \leqslant m, \forall i \in[2, t], x_{i} \in \mathbb{Z}^{+}}}\left\{x_{2} \cdot x_{3} \cdot \ldots \cdot x_{t}\right\} .
$$

For a fixed $t$ the product $x_{2} \ldots x_{t}$ in (6) obtains its maximum when $x_{i} \mathrm{~s}(i \geqslant 2)$ are as equal as possible (for all $i, j\left|x_{i}-x_{j}\right| \leqslant 1$ ), and their sum is equal to $m$. Let $\left\lfloor\frac{m}{n-1}\right\rfloor=s$, $\frac{m}{n-1}=s+\alpha$.

If $s \geqslant 3$ (which is equivalent to $\frac{m}{n-1} \geqslant 3$ ), let the maximum in (6) be achieved for some $t \leqslant n$ and let $x_{2}, \cdots, x_{t}$ be a collection of $x_{i}$ s that gives the maximum in (6). If $t<n$, then $s \geqslant 3$ implies that either for some $i \in[t], x_{i} \geqslant 5$, or for two different $i, j \in[t], x_{i}=x_{j}=4$. In the first case replacing $x_{i}$ by $x_{i}-2$ and setting $x_{t+1}=2$ gives a collection of $x_{i}$ 's with a bigger product. In the second case setting $x_{i}=x_{j}=3$ and $x_{t+1}=2$ increases the product of $x_{i}$ s. Hence, the maximum in (6) is achieved when $t=n$. For all $2 \leqslant i \leqslant n, x_{i}=s$ or $x_{i}=s+1$. Then the number of cycles in $G$ that pass through $v_{1}$ is at most

$$
\frac{\Delta}{2} x_{2} \ldots x_{n}=\frac{\Delta}{2} s^{(1-\alpha)(n-1)}(s+1)^{\alpha(n-1)}=\frac{\Delta}{2}\left(s^{1-\alpha}(s+1)^{\alpha}\right)^{n-1} .
$$

If $s<3$, let the maximum of (6) be achieved for some $2 \leqslant t \leqslant n$ and let $x_{2}, \cdots, x_{t}$ be the collection of $x_{i}$ s that gives the maximum in (6). Recall that for all $i, j\left|x_{i}-x_{j}\right| \leqslant 1$. If for two different $i, j \in[t] x_{i}=x_{j}>3$, then $m>6+3(t-2)=3 t$, and $s<3$ implies that $t<n$. Replacing $x_{i}$ by $x_{i}-1, x_{j}$ by $x_{j}-1$ and setting $x_{t+1}=2$ increases the product. Therefore, there is at most one $i$, such that $x_{i}=4$. If there is $i$ such that $x_{i}=1$, then replacing any $x_{j}(j \neq i)$ by $x_{j}+1$ and deleting $x_{i}$ increases the product. If for some $i, j, k \in$ $[t] x_{i}=x_{j}=x_{k}=2$, then replacing $x_{i}$ by $3, x_{j}$ by 3 and deleting $x_{k}$ increases the product. Therefore, $\left\{x_{2}, \ldots, x_{t}\right\} \in\{\{3,3, \ldots, 3,2,2\},\{3,3, \ldots, 3,4\},\{3,3, \ldots, 3,2\},\{3,3, \ldots, 3\}\}$. Then $x_{2} \ldots x_{t}$ is at most $3^{\frac{m}{3}}$, so the number of cycles that pass through $v_{1}$ is at most

$$
\frac{\Delta}{2} x_{2} \ldots x_{t} \leqslant \frac{\Delta}{2} 3^{\frac{m}{3}}
$$

Proof of Theorem 1. The proof is by mathematical induction on $n$.

Base case. If $n=2$, there is only one multigraph on $n$ vertices with $m$ edges - two vertices connected by $m$ edges. In this case $s=\frac{m}{n-1}=m$, and $G$ has $\max \left\{\left(\begin{array}{c}m \\ 2\end{array}\right), 0\right\}$ cycles, which is less than $\frac{3}{4} m(\sqrt[3]{3})^{m}$ (for the case $m<3$ ), and less than $\frac{3}{4} m \cdot m$ (for the case $m \geqslant 3$ ). Inductive step. Let $k \geqslant 3$ be an integer, and suppose that the statement of the theorem is proved for $n=k-1$. Let $G$ be a multigraph with $k$ vertices, $m$ edges and let $v_{1}$ be a vertex of maximal degree in $G$.

Case 1: Suppose that $\frac{m}{k-1}<3$.

If $\Delta(G) \leqslant 2$, then every edge is contained in at most one cycle, and every cycle contains at least two edges, so the number of cycles in $G$ is at most

$$
\frac{m}{2} \leqslant \frac{3}{4} \Delta(G) \cdot(\sqrt[3]{3})^{m}
$$


If $\Delta(G) \geqslant 3$, then the multigraph $G-v_{1}$ has at most $m-3$ edges, $\Delta\left(G-v_{1}\right) \leqslant \Delta(G)$ and $\frac{\left|E\left(G-v_{1}\right)\right|}{\left|V\left(G-v_{1}\right)\right|-1} \leqslant \frac{m}{k-1}<3$, therefore, by inductive assumption, the number of cycles in $G-v_{1}$ is at most $\frac{3}{4} \Delta(G) \cdot(\sqrt[3]{3})^{m-3}$. By Lemma 5 , the number of cycles that contain $v_{1}$ is at most $\frac{\Delta(G)}{2} \cdot(\sqrt[3]{3})^{m}$, therefore the total number of cycles in $G$ is at most

$$
\frac{\Delta(G)}{2} \cdot(\sqrt[3]{3})^{m}+\frac{3}{4} \Delta(G) \cdot(\sqrt[3]{3})^{m-3}=\frac{3}{4} \Delta(G) \cdot(\sqrt[3]{3})^{m}
$$

Case 2: Now we consider the case $\frac{m}{k-1} \geqslant 3$.

Let $s=\left\lfloor\frac{m}{k-1}\right\rfloor, \alpha=\frac{m}{k-1}-\left\lfloor\frac{m}{k-1}\right\rfloor$. Note that $\Delta\left(G-v_{1}\right) \leqslant \Delta(G)$ and let

$$
y=\frac{\left|E\left(G-v_{1}\right)\right|}{\left|V\left(G-v_{1}\right)\right|-1} \leqslant \frac{m}{k-1} .
$$

Note that the function

$$
f(x)=(\lfloor x\rfloor)^{1-x+\lfloor x\rfloor}(\lfloor x\rfloor+1)^{x-\lfloor x\rfloor}
$$

is non-decreasing on every interval $[a, a+1], a \in \mathbb{Z}_{\geqslant 0}$ (and hence on $\mathbb{R}^{+}$), therefore

$$
s^{1-\alpha}(s+1)^{\alpha} \geqslant f(3)=3 \text {. }
$$

If $y \geqslant 3$, then, by the induction hypothesis,

$$
\begin{aligned}
\left|E\left(G-v_{1}\right)\right| & \leqslant \frac{3}{4} \Delta(G)\left((\lfloor y\rfloor)^{1-y+\lfloor y\rfloor}(\lfloor y\rfloor+1)^{y-\lfloor y\rfloor}\right)^{k-2} \\
& \leqslant \frac{3}{4} \Delta(G)\left(s^{1-\alpha}(s+1)^{\alpha}\right)^{k-2} .
\end{aligned}
$$

If $y<3$, then $\left|E\left(G-v_{1}\right)\right|<3(k-2)$, and by the induction hypothesis

$$
\begin{aligned}
\left|E\left(G-v_{1}\right)\right| & \leqslant \frac{3}{4} \Delta(G)(\sqrt[3]{3})^{\left|E\left(G-v_{1}\right)\right|}<\frac{3}{4} \Delta(G)(\sqrt[3]{3})^{3(k-2)} \\
& =\frac{3}{4} \Delta(G) \cdot 3^{k-2} \leqslant \frac{3}{4} \Delta(G)\left(s^{1-\alpha}(s+1)^{\alpha}\right)^{k-2} .
\end{aligned}
$$

Hence, for any $y,\left|E\left(G-v_{1}\right)\right| \leqslant \frac{3}{4} \Delta(G)\left(s^{1-\alpha}(s+1)^{\alpha}\right)^{k-2}$, which together with Lemma 5 and (7) implies that

$$
\begin{aligned}
C(G) & =\frac{3 \Delta(G)}{4}\left(s^{1-\alpha}(s+1)^{\alpha}\right)^{k-2}+\frac{\Delta(G)}{2}\left(s^{1-\alpha}(s+1)^{\alpha}\right)^{k-1} \\
& \leqslant \frac{3 \Delta(G)}{4}\left(s^{1-\alpha}(s+1)^{\alpha}\right)^{k-1},
\end{aligned}
$$

which proves the inductive step and hence the theorem.

Proof of Corollary 2. Let $G$ be a graph with $n$ vertices and $m$ edges, such that $C(G)=$ $C(m)$. First, prove the statement of the corollary for the case when $\Delta(G) \leqslant 6$. Suppose that $\frac{m}{n-1} \geqslant 3$. Let $f(s, \alpha)=\left(s^{1-\alpha}(s+1)^{\alpha}\right)^{\frac{1}{s+\alpha}}$, then for any $s>0, f(s, \alpha)$ is monotone 
in $\alpha$ and $\max _{s \in \mathbb{Z}_{+}, \alpha \in[0,1)} f(s, \alpha)=\max _{s \in \mathbb{Z}_{+}} s^{\frac{1}{s}}=\sqrt[3]{3}$. This, together with Theorem 1, implies that for $s=\left\lfloor\frac{m}{n-1}\right\rfloor$ and $\alpha=\frac{m}{n-1}-\left\lfloor\frac{m}{n-1}\right\rfloor$

$$
C(m)=C(G)<\frac{3}{4} \Delta(G)\left(\left(s^{1-\alpha}(s+1)^{\alpha}\right)^{\frac{1}{s+\alpha}}\right)^{m} \leqslant 4.5(\sqrt[3]{3})^{m} .
$$

If $\frac{m}{n-1}<3$ then, by Theorem 1 ,

$$
C(m)=C(G)<\frac{3}{4} \Delta(G)(\sqrt[3]{3})^{m} \leqslant 4.5(\sqrt[3]{3})^{m}
$$

The proof for the case $\Delta(G) \geqslant 7$ goes by induction on $m$. Let $v$ be a vertex of degree at least 7 in $G$, then there exists an edge $e$, incident to $v$, such that there are at most $\frac{2}{7} C(G)$ cycles in $G$ that contain $e$. Hence $C(G) \leqslant \frac{5}{7} C(G-e)$, and by inductive hypothesis, $C(G-e) \leqslant 4.5(\sqrt[3]{3})^{m-1}$. Therefore

$$
C(G) \leqslant \frac{5}{7} 4.5(\sqrt[3]{3})^{m-1} \leqslant 4.5(\sqrt[3]{3})^{m}
$$

In Appendix A it is shown that extremal graphs for the function $C(m)$ have bounded degrees. Theorem 10 states that if $G$ is a graph with $m$ edges with $C(G)=C(m)$, then the maximum degree of $G$ is at most 11 .

\section{Example of a graph with $(1.37)^{m}$ cycles}

In this section we present an example of a graph with $m$ edges that has at least $(1.37)^{m}$ cycles. The previous best known bound is due to Alt, Fuchs and Kriegel [3] who provided an example of a planar graph with $1.34^{m}$ cycles.

For $n \geqslant 1$ let $H_{n}$ be the graph on $2 n+2$ vertices with

$$
\begin{gathered}
V\left(H_{n}\right)=\left\{u_{1}, u_{2}, \ldots, u_{n+1}, v_{1}, v_{2}, \ldots v_{n+1}\right\} \quad \text { and } \\
E\left(H_{n}\right)=\left\{u_{i} v_{j}: i, j \in[n+1],|i-j| \leqslant 1\right\} \cup\left\{u_{i} u_{i+1}: i \in[n]\right\} \cup\left\{v_{i} v_{i+1}: i \in[n]\right\} .
\end{gathered}
$$

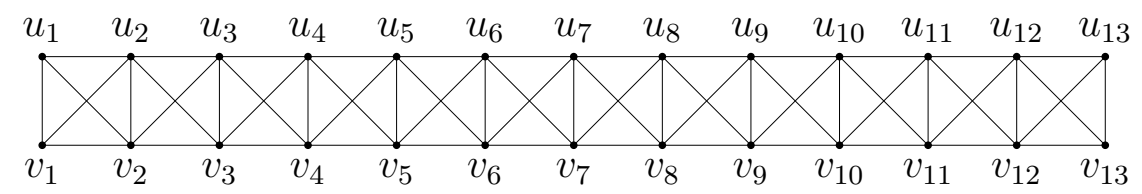

Figure 1: Graph $H_{12}$.

For $n \geqslant 1$ denote by $P(n)$ the number of paths from the vertex $u_{1}$ to the vertex $u_{n+1}$ in $H_{n}$. Note that $P(n)$ is also equal to the number of paths from $u_{1}$ to $v_{n+1}$ in $H_{n}$. 
Claim 6. For all $n \geqslant 2$

$$
P(n)=4 P(n-1)+4 P(n-2) .
$$

Proof sketch. The proof of the claim relies on an inductive argument and an observation that each path from $u_{1}$ to $u_{n+1}$ in $H_{n}$ corresponds to exactly one of the following paths:

- path from $u_{1}$ to $u_{n}$ in $H_{n-1}$ followed by the path $u_{n} u_{n+1}$ or by the path $u_{n} v_{n+1} u_{n+1}$.

- path from $u_{1}$ to $v_{n}$ in $H_{n-1}$ followed by the path $v_{n} u_{n+1}$ or by the path $v_{n} v_{n+1} u_{n+1}$.

- path from $u_{1}$ to $u_{n-1}$ in $H_{n-2}$ followed by the path $u_{n-1} u_{n} v_{n+1} v_{n} u_{n+1}$ or by the path $u_{n-1} v_{n} v_{n+1} u_{n} u_{n+1}$.

- path from $u_{1}$ to $v_{n-1}$ in $H_{n-2}$ followed by the path $v_{n-1} u_{n} v_{n+1} v_{n} u_{n+1}$ or by the path $v_{n-1} v_{n} v_{n+1} u_{n} u_{n+1}$.

Solving the recurrence relation leads to the inequality

$$
P(n) \geqslant(2+2 \sqrt{2})^{n} .
$$

Define the graph $G_{n}$ by identifying vertices $u_{1}$ and $u_{n+1}$ in $H_{n}$. Then $G_{n}$ has $2 n+1$ vertices, $m=5 n+1$ edges and

$$
C\left(G_{n}\right) \geqslant P(n) \geqslant(2+2 \sqrt{2})^{n} .
$$

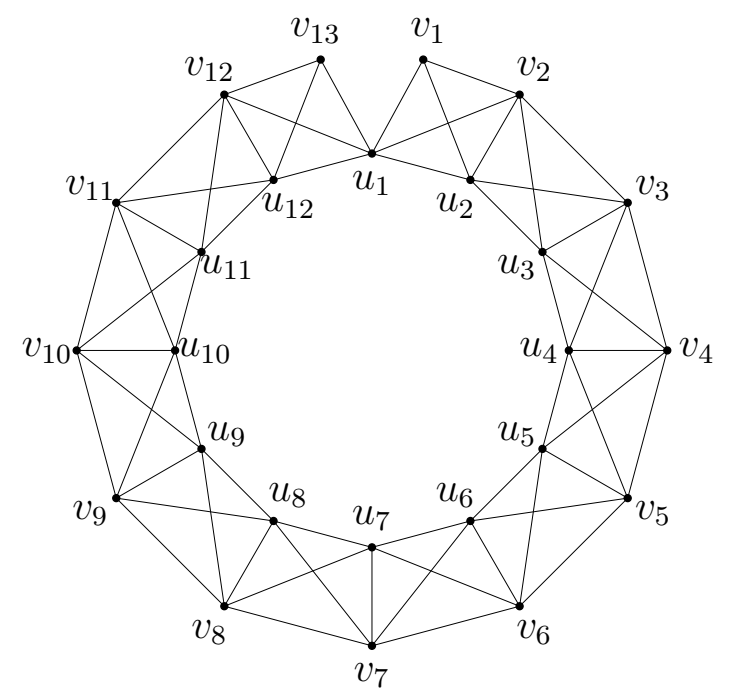

Figure 2: $G_{12}$ with 25 vertices and 61 edges.

For an integer $m$ let graph $G$ be obtained from $G_{\left\lfloor\frac{m-1}{5}\right\rfloor}$ by adding $\left(m-5\left\lfloor\frac{m-1}{5}\right\rfloor-1\right)$ edges. Then $G$ has $m$ edges and for $m$ large enough

$$
C(G) \geqslant C\left(G_{\left\lfloor\frac{m-1}{5}\right\rfloor}\right) \geqslant(2+2 \sqrt{2})^{\left\lfloor\frac{m-1}{5}\right\rfloor} \geqslant(2+2 \sqrt{2})^{\frac{m}{5}-1}>1.37^{m} .
$$




\section{Maximum number of cycles in multigraphs}

The problems of maximizing the number of cycles with fixed number of edges or fixed average degree can also be considered for multigraphs. Using the techniques presented in this paper, the authors can prove the following two results.

Theorem 7. Let $G$ be a multigraph that has the maximum number of cycles among all the multigraphs with $n \geqslant 2$ vertices and $m \geqslant 3$ edges. Let $\left\lfloor\frac{m}{n-1}\right\rfloor=s, \alpha=\frac{m}{n-1}-s$. If $\frac{m}{n-1} \geqslant 3$, then

$$
\frac{8}{27} s\left(s^{1-\alpha}(s+1)^{\alpha}\right)^{n-1} \leqslant C(G) \leqslant \frac{3}{4} \Delta(G)\left(s^{1-\alpha}(s+1)^{\alpha}\right)^{n-1} .
$$

If $\frac{m}{n-1} \leqslant 3$, then

$$
4(\sqrt[3]{3})^{m-4} \leqslant C(G)<\frac{3}{4} \Delta(G) \cdot(\sqrt[3]{3})^{m} .
$$

The upper bounds in Theorem 7 follow from Theorem 1. For the lower bounds, define $C_{n, m}$ to be the multigraph obtained from the cycle $C_{n}$ by replacing each of some $m-\left\lfloor\frac{m}{n}\right\rfloor n$ consecutive edges with edges of multiplicity $\left\lfloor\frac{m}{n}\right\rfloor+1$ and the rest $\left\lfloor\frac{m}{n}\right\rfloor n-m+n$ edges with edges of multiplicity $\left\lfloor\frac{m}{n}\right\rfloor$. The lower bound in the first case is achieved by the graph $C_{n, m}$. The lower bound in the second case is achieved by the graph $C_{\left\lfloor\frac{m+1}{3}\right\rfloor, m}$ with extra

$n-\left\lfloor\frac{m+1}{3}\right\rfloor$ isolated vertices. For a detailed proof of Theorem 7 we refer an interested reader to $[4,14]$.

Theorem 8. Let $G$ be a multigraph with $m \geqslant 3$ edges that has the maximum number of cycles among all the multigraphs with $m$ edges. Then

$$
\frac{9}{10}(\sqrt[3]{3})^{m}<4(\sqrt[3]{3})^{m-4} \leqslant C(G) \leqslant 4.5(\sqrt[3]{3})^{m}
$$

The upper bound in Theorem 8 can be obtained by repeating the argument of Corollary 2 and a version of Theorem 10, modified for multigraphs. The example for the lower bound is the same as for the second case of Theorem 7 .

Theorems 7 and 8 answer both questions for multigraphs up to a constant factor. The authors believe that for $m \geqslant 9$ the graph $C_{\left\lfloor\frac{m+1}{3}\right\rfloor, m}$ has the most cycles among all multigraphs with $m$ edges.

\section{Concluding remarks}

Theorem 1 gives an upper bound for the number of cycles in a graph $G$ with $n$ vertices and $m$ edges. For a graph $G$ with $n$ vertices and average degree $d \geqslant 6$, Theorem 1 and AM-GM imply

$$
C(G) \leqslant 3 \Delta(G)\left(\frac{d}{2}\right)^{n}
$$


For $d=\Omega(\ln n)$, let $G$ be a random graph $G(n, p)$ with $p=\frac{d}{n-1}$. Glebov and Krivelevich [8] proved that the number of hamiltonian cycles in $G$ is a.a.s. at least $\left(\frac{d}{e}\right)^{n}(1+o(1))^{n}$. It was shown in $[4,14]$ that, provided $d \rightarrow \infty$, a random $G(n, m)$ graph has $\left(\frac{d}{e}\right)^{n}(1+o(1))^{n}$ cycles in expectation. Therefore, if $G$ is a graph with the maximal number of cycles among all graphs with $n$ vertices and average degree $d$ (with $d \rightarrow \infty$ ), then for $n$ large enough

$$
\left(\frac{d}{e}\right)^{n}(1+o(1))^{n} \leqslant C(G) \leqslant(1+o(1))^{n}\left(\frac{d}{2}\right)^{n} .
$$

In an earlier version of this paper, we conjectured that $C(G) \leqslant\left((1+o(1)) \frac{d}{e}\right)^{n}$. This was shown to be true by Noel [12], who used Bregman's theorem to show that

$$
C(G) \leqslant(d+1) !^{n /(d+1)}=(1+o(1))^{n}(d / e)^{n} .
$$

We note that the bound in Theorem 1 is exponentially better than the one of Noel for the case when $d<9.2411$.

As mentioned in the introduction, Theorem 2 and the result of Section 3 imply that

$$
1.37^{m} \leqslant C(m) \leqslant 1.443^{m}
$$

Király [9] conjectured that $C(m)<1.4^{m}$. The upper bound in Corollary 2 is $4.5(\sqrt[3]{3})^{m}$, which inspired the following conjecture.

Conjecture 9. For sufficiently large $m$, there exists a graph $G$ with $m$ edges and at least $(1+o(1))^{m}(\sqrt[3]{3})^{m}$ cycles.

\section{Acknowledgements}

We would like to thank Karen Gunderson and Jamie Radcliffe for helpful discussions and Jonathan Noel for informing us about his observations. We would also like to thank David Gunderson for valuable comments and suggestions.

\section{Added in proof:}

After submission of this paper Dvořák, Morrison, Noel, Norin and Postle [6] have substantially improved Theorem 1 and disproved Conjecture 9. Their proof is based on bounding the number of paths between two vertices in a graph by a function of degree sequence that is much more intricate, compared to the one used in this paper.

During the preparation of this paper, Morrison, Roberts and Scott [11] have considered the problem of maximising $C(G)$ for the class of $H$-free graphs. For the class of $H$-free graphs they obtain bounds that are exponentially better than the ones in Theorem 1 , provided $m$ is close to the Turán number of $H$. 


\section{References}

[1] W. Ahrens, Ueber das Gleichungssystem einer Kirchhoff'schen galvanischen Stromverzweigung, Math. Ann. 49 (1897), 311-324 (German).

[2] R. E. L. Aldred and C. Thomassen, On the maximum number of cycles in a planar graph, J. Graph Theory 57 (2008), 255-264.

[3] H. Alt, U. Fuchs, and K. Kriegel, On the number of simple cycles in planar graphs, Combin. Probab. Comput. 8 (1999), 397-405.

[4] A. Arman, Maximum number of cycles in graphs and multigraphs. Ph.D. thesis, University of Manitoba, http://hdl.handle.net/1993/33030 (2019).

[5] A. Arman, D. S. Gunderson, and S. Tsaturian, Triangle-free graphs with the maximum number of cycles, Discrete Math. 339 (2016), 699-711.

[6] Z. Dvořák, N. Morrison, J. A. Noel, S. Norin, and L. Postle, Bounding the number of cycles in a graph in terms of its degree sequence, preprint available at arXiv:1907.12091, accessed 23 September 2019.

[7] R. C. Entringer and P. J. Slater, On the maximum number of cycles in a graph, Ars Combin. 11 (1981), 289-294.

[8] R. Glebov and M. Krivelevich, On the number of Hamilton cycles in sparse random graphs, SIAM J. Discrete Math. 27 (2013), 27-42.

[9] Z. Király, Maximum number of cycles and hamiltonian cycles in sparse graphs, 2009. Technical report, http://web.cs.elte.hu/egres/tr/egres-09-03.pdf, accessed 23 September 2019.

[10] P. Mateti and N. Deo, On algorithms for enumerating all circuits of a graph, SIAM J. Comput. 5 (1976), 90-99.

[11] N. Morrison, A. Roberts, and A. Scott, Maximising the number of cycles in graphs with forbidden subgraphs, preprint available at arXiv:1902.08133, accessed 23 May 2019.

[12] J. A. Noel, Cycle counting via the permanent. communication via email, 13 February 2019.

[13] Y. Shi, The number of cycles in a Hamilton graph, Discrete Math. 133 (1994), 249257.

[14] S. Tsaturian, Problems in extremal graph theory and Euclidean Ramsey theory. Ph.D. thesis, University of Manitoba, http://hdl.handle.net/1993/33849 (2019). 


\section{Appendix A Maximal degree of graphs with $C(m)$ cycles}

Recall that, for $m \in \mathbb{Z}^{+}, C(m)$ is the maximum number of cycles in a graph with $m$ edges.

Theorem 10. If $G$ is a graph with $m$ edges such that $C(G)=C(m)$, then $\Delta(G) \leqslant 11$.

The proof of Theorem 10 relies on the following two lemmas.

Lemma 11. Let $k \geqslant 6$ be a positive integer. For $1 \leqslant i<j \leqslant k$, let $w_{i, j}$ be a non-negative real number, and let $S=\sum_{1 \leqslant i<j \leqslant k} w_{i, j}$. Then there exists a 6 -element set $D \subseteq[k]$ such that

$$
\sum_{\substack{1 \leqslant i<j \leqslant k \\ i \notin D, j \notin D}} w_{i, j} \geqslant\left(1-\frac{6(2 k-7)}{k(k-1)}\right) S .
$$

Proof. The proof relies on an averaging argument. For each $i \in[k]$ set $w_{i}=\sum_{j \in[k], j \neq i} w_{i, j}$. Note that

$$
\sum_{i \in[k]} w_{i}=2 S
$$

Let $X$ be a collection of all 6 -element subsets of $[k]$. For $D \in X$ let

$$
\begin{aligned}
S(D) & =\sum_{\substack{1 \leqslant i<j \leqslant k \\
i \notin D, j \notin D}} w_{i, j} \\
& =S-\sum_{i \in D} w_{i}+\sum_{i, j \in D, i<j} w_{i, j} .
\end{aligned}
$$

Let $\overline{S(D)}$ be the average of $S(D)$ over all $D \in X$, then

$$
\begin{aligned}
\overline{S(D)} & =\frac{\sum_{D \in X}\left(S-\sum_{i \in D} w_{i}+\sum_{i, j \in D, i<j} w_{i, j}\right)}{\left(\begin{array}{c}
k \\
6
\end{array}\right)} \\
& =S-\frac{\left(\begin{array}{c}
k-1 \\
5
\end{array}\right) \cdot 2 S}{\left(\begin{array}{l}
k \\
6
\end{array}\right)}+\frac{\left(\begin{array}{c}
k-2 \\
4
\end{array}\right) \cdot S}{\left(\begin{array}{l}
k \\
6
\end{array}\right)} \\
& =\left(1-\frac{6(2 k-7)}{k(k-1)}\right) S .
\end{aligned}
$$

There exists $D \in X$, such that $S(D) \geqslant \overline{S(D)}$, i.e.

$$
\sum_{\substack{1 \leqslant i<j \leqslant k \\ i \notin D, j \notin D}} w_{i, j} \geqslant\left(1-\frac{6(2 k-7)}{k(k-1)}\right) S .
$$


Lemma 12. Let $k \geqslant 2$ be a positive integer. For $1 \leqslant i<j \leqslant k$, let $w_{i, j}$ be a non-negative real number, and let $S=\sum_{1 \leqslant i<j \leqslant k} w_{i, j}$. Then there exists a partition $A_{1} \cup A_{2} \cup A_{3} \cup A_{4}=$ $[k]$, such that

$$
\sum_{1 \leqslant l<m \leqslant 4} \sum_{\substack{i \in A_{l} \\ j \in A_{m}}} w_{i, j} \geqslant\left(\frac{3 k^{2}-4}{4 k(k-1)}\right) S .
$$

Proof. For all $l \in[4]$ let $a_{l}=\left\lfloor\frac{k+l-1}{4}\right\rfloor$ (note that $a_{1}+a_{2}+a_{3}+a_{4}=k$ ). Let $X$ be the collection of all ordered quadruples $\left(A_{1}, A_{2}, A_{3}, A_{4}\right)$, such that $\pi=A_{1} \cup A_{2} \cup A_{3} \cup A_{4}$ is a partition of $[k]$ and for all $l \in[4],\left|A_{l}\right|=a_{l}$. Note that

$$
|X|=\frac{k !}{a_{1} ! a_{2} ! a_{3} ! a_{4} !} .
$$

For $p=\left(A_{1}, A_{2}, A_{3}, A_{4}\right) \in X$ define

$$
S(p)=\sum_{1 \leqslant l<m \leqslant 4} \sum_{\substack{i \in A_{l} \\ j \in A_{m}}} w_{i, j}=S-\sum_{l \in[4]} \sum_{\substack{i<j \\ i, j \in A_{l}}} w_{i, j} .
$$

Let $\overline{S(p)}$ be the average of $S(p)$ over all possible choices of $p$.

$$
\begin{aligned}
\overline{S(p)} & =\frac{\sum_{p \in X}\left(S-\sum_{l \in[4]} \sum_{i, j \in A_{l}, i<j} w_{i, j}\right)}{|X|} \\
& =S-\frac{\sum_{l \in[4]} \sum_{1 \leqslant i<j \leqslant k} \sum_{p \in X: i, j \in A_{l}} w_{i, j}}{|X|}
\end{aligned}
$$

Note that for any choice of $l \in[4]$ and any choice of $i, j$, such that $1 \leqslant i<j \leqslant k$ there are exactly

$$
\frac{(k-2) !\left(a_{l}\right)\left(a_{l}-1\right)}{a_{1} ! a_{2} ! a_{3} ! a_{4} !}
$$

quadruples $p \in X$, such that $i, j \in A_{l}$. Then,

$$
\begin{aligned}
\overline{S(p)} & =S-\left(\sum_{l \in[4]} \sum_{1 \leqslant i<j \leqslant k} \frac{(k-2) !\left(a_{l}\right)\left(a_{l}-1\right)}{a_{1} ! a_{2} ! a_{3} ! a_{4} !} w_{i, j}\right) /|X| \\
& =S-\left(\sum_{l \in[4]} \frac{(k-2) !\left(a_{l}\right)\left(a_{l}-1\right)}{a_{1} ! a_{2} ! a_{3} ! a_{4} !} \cdot S\right) \cdot \frac{1}{|X|} \\
& =S-\left(\sum_{l \in[4]} \frac{\left\lfloor\frac{k+l-1}{4}\right\rfloor\left(\left\lfloor\frac{k+l-1}{4}\right\rfloor-1\right)}{k(k-1)}\right) \cdot S \\
& \geqslant S\left(1-\frac{(k-2)^{2}}{4 k(k-1)}\right) .
\end{aligned}
$$


There exists a $p=\left(A_{1}, A_{2}, A_{3}, A_{4}\right) \in X$, such that $S(p) \geqslant \overline{S(p)}$, therefore the partition $A_{1} \cup A_{2} \cup A_{3} \cup A_{4}$ satisfies the statement of Lemma 12 .

Proof of Theorem 10. Let $m$ be a positive integer and $G$ be a graph with $m$ edges. To prove Theorem 10, it is sufficient to prove that if $\Delta(G) \geqslant 12$, then there is a graph $H$ with $m$ edges and with $C(H)>C(G)$.

Let $\Delta(G) \geqslant 12$ and $u$ be a vertex of maximal degree in $G$. Let $N(u)=\left\{u_{1}, u_{2}, \ldots, u_{k}\right\}$ be the neighbourhood of $u$ (note that $k \geqslant 12$ ). For $1 \leqslant i<j \leqslant k$, define $w_{i, j}$ to be the number of paths from the vertex $u_{i}$ to the vertex $u_{j}$ in the graph $G-u$. Then the number of cycles in graph $G$ that pass through vertex $u$ is $S=\sum_{1 \leqslant i<j \leqslant k} w_{i, j}$. By Lemma 11, there is a 6 -element set $D=\left\{i_{1}, i_{2}, \ldots, i_{6}\right\}$, such that

$$
\sum_{\substack{1 \leqslant i<j \leqslant k \\ i \notin D, j \notin D}} w_{i, j} \geqslant\left(1-\frac{6(2 k-7)}{k(k-1)}\right) S .
$$

Suppose, upon re-indexing, that $D=\{k-5, k-4, \ldots, k-1, k\}$. Applying Lemma 12 to the collection of real numbers $w_{i, j}$ with $1 \leqslant i<j \leqslant k-6$ gives a partition $A_{1} \cup A_{2} \cup A_{3} \cup A_{4}=[k-6]$ with

$$
\sum_{1 \leqslant l<m \leqslant 4} \sum_{\substack{i \in A_{l} \\ j \in A_{m}}} w_{i, j} \geqslant\left(\frac{3(k-6)^{2}-4}{4(k-6)(k-7)}\right)\left(1-\frac{6(2 k-7)}{k(k-1)}\right) S .
$$

For $i \in[4]$, let $U_{i}=\left\{u_{j}: j \in A_{i}\right\}$. Construct a graph $H$ by deleting $u$ and all of the edges incident to $u$, adding four new vertices $v_{1}, v_{2}, v_{3}, v_{4}$, then for all $1 \leqslant i \leqslant 4$ adding edges from $v_{i}$ to each vertex of $U_{i}$, and for all $1 \leqslant i<j \leqslant 4$ adding edges $v_{i} v_{j}$ (see Figure 3). Then $|E(H)|=|E(G)|$.

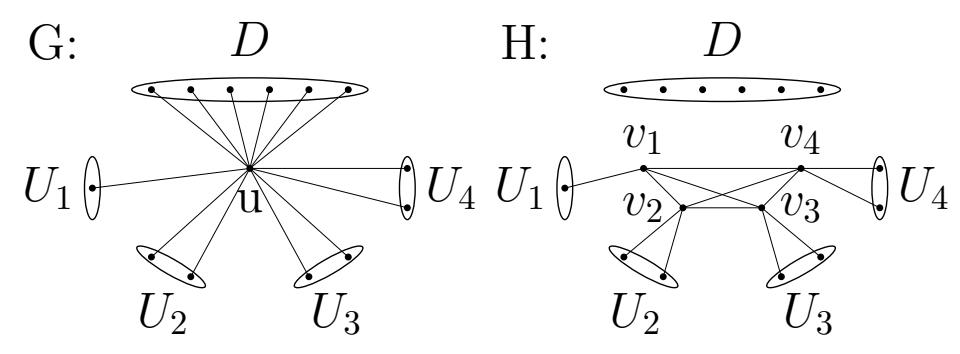

Figure 3: Constructing graph $H$ from $G$.

To count the number of cycles in $H$, note the following:

- Every cycle in $G$ that does not pass through the vertex $u$ is still a cycle in $H$. There are $C(G)-S$ such cycles. 
- Let $C$ be a cycle in $G$ that for some $1 \leqslant i<j \leqslant k-6$ contains a path $u_{i} u u_{j}$. If for some $l \in[4] u_{i}$ and $u_{j}$ are in the same class $U_{l}$, then $C$ corresponds to the cycle in $H$ that uses the path $u_{i} v_{l} u_{j}$ instead of $u_{i} u u_{j}$. In the case if $u_{i} \in U_{l}$ and $u_{j} \in U_{m}$ for some $1 \leqslant l<m \leqslant 4$, cycle $C$ corresponds to the cycle that uses the path $u_{i} v_{l} v_{m} u_{j}$ instead of $u_{i} u u_{j}$. By (10), there are at least

$$
\left(1-\frac{6(2 k-7)}{k(k-1)}\right) S
$$

cycles in $G$ that use path $u_{i} u u_{j}$ with $u_{i}, u_{j} \in N(u) \backslash D$.

- Every cycle in $G$ that for some $i \in A_{l}$ and $j \in A_{m}$ with $l \neq m$ contains a path $u_{i} u u_{j}$ gives rise to additional 4 cycles in $H$ (except the one containing $u_{i} v_{l} v_{m} u_{j}$ ). For example, if $l=1, m=2$ the four new cycles contain paths $u_{i} v_{1} v_{3} v_{2} u_{j}, u_{i} v_{1} v_{4} v_{2} u_{j}$, $u_{i} v_{1} v_{3} v_{4} v_{2} u_{j}$ and $u_{i} v_{1} v_{4} v_{3} v_{2} u_{j}$ instead of $u_{i} u u_{j}$. According to (11), there are at least

$$
\left(\frac{3(k-6)^{2}-4}{4(k-6)(k-7)}\right)\left(1-\frac{6(2 k-7)}{k(k-1)}\right) S=\left(\frac{3 k^{2}-36 k+104}{4 k(k-1)}\right) S
$$

cycles in $G$ that for some $i \in A_{l}$ and $j \in A_{m}$ with $l \neq m$ pass through a path $u_{i} u u_{j}$.

- There are 7 new cycles in $H$ spanned by the vertices $v_{1}, v_{2}, v_{3}, v_{4}$.

By all of the observations above, the number of cycles in $H$ is

$$
\begin{aligned}
C(H) & \geqslant C(G)-S+\left(1-\frac{6(2 k-7)}{k(k-1)}\right) S+4\left(\frac{3 k^{2}-36 k+104}{4 k(k-1)}\right) S+7 \\
& >C(G)+7+S\left(\frac{3(k-4)(k-12)}{k(k-1)}\right) \\
& >C(G) .
\end{aligned}
$$

Therefore, $H$ has more cycles than $G$.

Note, that for $m=7$ the graphs that have the most cycles are $K_{4}$ plus an edge and $K_{4}$ with one edge replaced by a path of length two. In the first case minimum degree is one, in the second case - two.

The authors can also prove the following theorem (for the proof, see [4]).

Theorem 13. If $m>7$ and $G$ is a connected graph with $C(G)=C(m)$, then $\delta(G) \geqslant 3$. 